\title{
SYNTHESIS, HIRSHFELD SURFACE ANALYSES AND MAGNETISM OF A $1 D$ Mn(II) COMPLEX
}

\author{
Lu Lu ${ }^{1 *}$, Jun Wang ${ }^{1}$, Wei-Ping $\mathrm{Wu}^{1}, \mathrm{Xi}-\mathrm{Ren} \mathrm{Wu}^{2 *}$ and Abhinav Kumar ${ }^{3 *}$ \\ ${ }^{1}$ School of Chemistry and Pharmaceutical Engineering, Sichuan University of Science \& \\ Engineering, Zigong 643000, China \\ ${ }^{2}$ School of Pharmacy, Guangdong Medical University, Dongguan, 523808, China \\ ${ }^{3}$ Department of Chemistry, Faculty of Science, University of Lucknow, Lucknow 226007 , India
}

(Received April 8, 2015; revised September 17, 2015)

\begin{abstract}
A new Mn-based complex of $\left\{\left[\mathrm{Mn}(\mathrm{L})_{2}(\mathrm{mi})\right] \cdot \mathrm{H}_{2} \mathrm{O}\right\}_{\mathrm{n}}(\mathbf{1})(\mathrm{HL}=p$-hydroxy phenylacetic acid; $\mathrm{mi}=$ 1,1'-(1,4-butanediyl)bis(imidazole)), has been synthesized and structurally characterized. Single-crystal X-ray analyses reveal that compound 1 has a dinuclear $\mathrm{Mn}$ (II) unit linking by four carboxylate groups. The bridging Ndonor ligand with mi links the Mn(II) centers into a 1D double chain. The detailed analyses of Hirschfeld surface and fingerprint plots provide insight into the nature of non-covalent interactions in the title compound. Furthermore, an attempt was made to explain the magnetic property of compound $\mathbf{1}$ using atoms in molecule (AIM) theory.
\end{abstract}

KEY WORDS: Supramolecular architecture, Hirschfeld surface analysis, Magnetism

\section{INTRODUCTION}

Coordination polymers (CPs) have attracted intense attention in recent years because of their intriguing structures and potential applications as functional materials [1-3]. Flexible carboxylic acids are good candidates for the construction of new coordination polymers as the carboxyl groups can form $\mathrm{C}-\mathrm{O}-\mathrm{M}-\mathrm{O}$ cyclic mode with central metal ions, thus, improving the stability of transition metal complexes [4]. Furthermore, such types of the carboxylate and carboxyl groups are always actively involved in H-bonding interactions, which results in many interesting structures with higher dimensions [5-10].

On the other hands, most of MOFs with mixed-ligands have been reported [11-13]. The combination of different ligands can result in greater modulation of structural frameworks than single ligand. Hence, mixed-ligands are undoubtedly a good choice for the construction of new polymeric structures. In particular, coordination compounds with flexible ligands exhibit more complex structural peculiarity due to the characteristics of flexible ligands, namely, the configurations of functional groups were changeful. Therefore, we selected two flexible ligands $\mathrm{HL}$ and $\mathrm{mi}$ as the primary ligands for coordination to the Mn salt to synthesize the compound. We report here the synthesis of a dinuclear $\mathrm{Mn}(\mathrm{II})$ complex with mixed co-ligands. The compound 1 was characterized by single crystal X-ray diffraction. Subsequently, the compound was analyzed for their crystal packing. In order to evaluate the nature and energetic associated with intermolecular interactions in the crystal packing, the detailed analyses of Hirschfeld surface and fingerprint plots calculations were performed. The total lattice energy is partitioned into the corresponding Columbic, polarization, dispersion and repulsion energies. Furthermore, an attempt was made to explain the magnetic property of compound $\mathbf{1}$ using atoms in molecule (AIM) theory.

\section{Materials and method}

\section{EXPERIMENTAL}

All reagents were purchased from commercial sources and used as received. IR spectra were recorded with a Perkin-Elmer Spectrum One spectrometer in the region $4000-400 \mathrm{~cm}^{-1}$ using

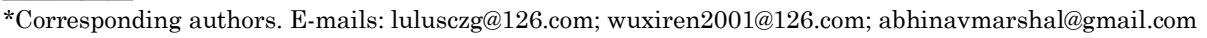


$\mathrm{KBr}$ pellets. TGA was carried out with a Metter-Toledo TA 50 in dry dinitrogen $\left(60 \mathrm{~mL} \cdot \mathrm{min}^{-1}\right)$ at a heating rate of $5{ }^{\circ} \mathrm{C} \mathrm{min}^{-1}$. X-ray powder diffraction (XRPD) data were recorded on a Rigaku RU200 diffractometer at $60 \mathrm{KV}, 300 \mathrm{~mA}$ for $\mathrm{Cu} K_{\alpha}$ radiation $(\lambda=1.5406 \AA)$, with a scan speed of $2{ }^{\circ} \mathrm{C} / \mathrm{min}$ and a step size of $0.013^{\circ}$ in $2 \theta$. Magnetic susceptibility data of powdered sample restrained in parafilm were measured on Oxford Maglab 2000 magnetic measurement system in the temperature range $300-1.8 \mathrm{~K}$ and at field of $1 \mathrm{KOe}$.

\section{X-Ray crystallography}

Single crystal X-ray diffraction analysis of the title compound was carried out on a Bruker SMART APEX II CCD diffractometer equipped with a graphite monochromated MoKa radiation $(\lambda=0.71073 \AA)$ by using $\phi / \omega$ scan technique at room temperature. Data were processed using the Bruker SAINT package and the structures solution and the refinement procedure was performed using SHELX-97 [14]. The structure was solved by direct methods and refined by full-matrix least-squares fitting on $\mathrm{F}^{2}$. The hydrogen atoms of organic ligands were placed in calculated positions and refined using a riding on attached atoms with isotropic thermal parameters 1.2 times those of their carrier atoms. The hydrogen atoms of lattice water molecule in compound 1 were located using the different Fourier method. Table 1 shows crystallographic data of $\mathbf{1}$. Selected bond distances and bond angles are listed in Table 2. CCDC: 1044526.

Table 1. Crystal data and structure refinement information for compound $\mathbf{1 .}$

\begin{tabular}{|l|l|}
\hline Formula weight & 565.48 \\
\hline Crystal system & Triclinic \\
\hline Space group & $P$-1 \\
\hline Crystal color & Colorless \\
\hline$a, \AA$ & $10.8106(15)$ \\
\hline$b, \AA$ & $11.0003(15)$ \\
\hline$c, \AA$ & $12.945(2)$ \\
\hline$\alpha,^{\circ}$ & $114.089(14)$ \\
\hline$\beta,^{\circ}$ & $106.270(13)$ \\
\hline$\gamma,^{\circ}$ & $95.671(11)$ \\
\hline$V, \AA^{3}$ & $1308.5(4)$ \\
\hline$Z$ & 2 \\
\hline$\rho_{\text {calMn }}$ g/cm ${ }^{3}$ & 1.435 \\
\hline$\mu, m^{-1}$ & 0.557 \\
\hline$F(000)$ & 590 \\
\hline$\theta$ Range, deg & $2.53-25.30$ \\
\hline Reflection collected & 8906 \\
\hline Independent reflections $\left(R_{\text {int }}\right)$ & 0.0533 \\
\hline Reflections with $I>2 \sigma(I)$ & 3009 \\
\hline Number of parameters & 345 \\
\hline$R_{1}, w R_{2}(I>2 \sigma(I))^{* *}$ & $0.0606,0.1375$ \\
\hline$R_{1}, w R_{2}(\text { all data })^{* *}$ & $0.1013,0.1703$ \\
\hline
\end{tabular}

$* R=\sum\left(F_{\mathrm{o}}-F_{\mathrm{c}}\right) / \sum\left(\mathrm{F}_{\mathrm{o}}\right), * * w R_{2}=\left\{\sum\left[w\left(F_{\mathrm{o}}^{2}-F_{\mathrm{c}}{ }^{2}\right] / \sum\left(F_{\mathrm{o}}^{2}\right)^{2}\right\}^{1 / 2}\right.$.

\section{Hirshfeld surface analysis}

Molecular Hirshfeld surfaces [15] in the crystal structure were constructed on the basis of the electron distribution calculated as the sum of spherical atom electron densities $[16,17]$. For a given crystal structure and a set of spherical atomic densities, the Hirshfeld surface is unique [16]. The 
normalized contact distance $\left(d_{\text {norm }}\right)$ based on both $d_{\mathrm{e}}$ and $d_{\mathrm{i}}$ (where $d_{\mathrm{e}}$ is distance from a point on the surface to the nearest nucleus outside the surface and $d_{\mathrm{i}}$ is distance from a point on the surface to the nearest nucleus inside the surface) and the vdW radii of the atom, as given by Eq. 1 enables identification of the regions of particular importance to intermolecular interactions. The combination of $d_{\mathrm{e}}$ and $d_{\mathrm{i}}$ in the form of two-dimensional (2D) fingerprint plot [18, 19] provides a summary of intermolecular contacts in the crystal [15]. The Hirshfeld surfaces mapped with $d_{\text {norm }}$ and 2D fingerprint plots were generated using the Crystal-Explorer 2.1 [19]. Graphical plots of the molecular Hirshfeld surfaces mapped with $d_{\text {norm }}$ used a red-white-blue colour scheme, where red highlight shorter contacts, white represents the contact around vdW separation, and blue is for longer contact. Additionally, two further coloured plots representing shape index and curvedness based on local curvatures are also presented in this paper [20].

$d_{\text {norm }}=\frac{d_{\mathrm{i}}-r_{\mathrm{i}}^{\mathrm{vdW}}}{r_{\mathrm{i}}^{\mathrm{vdW}}}+\frac{d_{\mathrm{e}}-r_{\mathrm{e}}^{\mathrm{vdW}}}{r_{\mathrm{e}}^{\mathrm{vdW}}}$

Table 2. Selected bond distances $(\AA)$ and angles (deg) of structure 1.

\begin{tabular}{|l|l|l|l|}
\hline Bond & $d, \AA$ & Bond & $d, \AA$ \\
\hline Mn1-O1 & $2.087(3)$ & Mn1-O2A & $2.197(3)$ \\
\hline Mn1-O4 & $2.223(3)$ & Mn1-N1 & $2.244(3)$ \\
\hline Mn1-N4A & $2.247(3)$ & & \\
\hline Angle & $\omega, \mathrm{deg}$ & Angle & $\omega$, deg \\
\hline O1-Mn1-O2A & $123.34(19)$ & O1-Mn1-O4 & $93.63(19)$ \\
\hline O2A-Mn1-O4 & $142.91(13)$ & O1-Mn1-N1 & $94.79(14)$ \\
\hline O2A-Mn1-N1 & $90.24(12)$ & O4-Mn1-N1 & $89.37(12)$ \\
\hline O1-Mn1-N4A & $92.77(14)$ & O2A-Mn1-O4A & $85.63(12)$ \\
\hline O4-Mn1-N4A & $90.11(12)$ & N1-Mn1-N4A & $172.43(11)$ \\
\hline Symmetric code: (A) x, y, z. & \\
\hline
\end{tabular}

Synthesis of the complex $\left\{\left[\mathrm{Mn}(\mathrm{L})_{2}(\mathrm{mi})\right] \cdot \mathrm{H}_{2} \mathrm{O}\right\}_{n}(\mathbf{1})$

A mixture of $\mathrm{Mn}\left(\mathrm{NO}_{3}\right)_{2} \cdot 4 \mathrm{H}_{2} \mathrm{O}(0.0410 \mathrm{~g})$, $\mathrm{HL}(0.0116 \mathrm{~g}), \mathrm{mi}(0.0350 \mathrm{~g}), \mathrm{CH}_{3} \mathrm{OH}(5 \mathrm{~mL})$ and deionised water $(5 \mathrm{~mL})$ was stirred for $30 \mathrm{~min}$ in air. The resulting solution was kept at room temperature for one week, the crystals formed were filtered off, washed with water and dried in air. $\mathrm{C}_{26} \mathrm{H}_{30} \mathrm{MnN}_{4} \mathrm{O}_{7}$ (565.48). Calcd: C, 55.22; H, 5.35; N, 9.91. Found C, 55.10; H, 5.21; N, 9.87. IR $\left(\mathrm{KBr}, \mathrm{cm}^{-1}\right)$ : 3225(vs); 2964(m);2600(v); 1549(v); 1435(vs); 1315(v); 1212(v); 1092(v): 1007(v); 870(m); 802(v); 625(v): 551(m).

\section{RESULTS AND DISCUSSION}

\section{$\left\{\left[\mathrm{Mn}(\mathrm{L})_{2}(\mathrm{mi})\right] \cdot \mathrm{H}_{2} \mathrm{O}\right\}_{n}(\mathbf{1})$}

The results of crystallographic analysis revealed that the asymmetric unit of complex 1 contains one $\mathrm{Mn}$ (II) atom, two L anions, one mi ligand, and one lattice water molecule. As shown in Figure 1, the L ligand adopts two coordination modes. Two $\mathrm{O}$ atoms of one carboxylate group exhibit a bismonodentate coordination mode to bridge two Mn centers, whereas the other carboxylate group coordinates to one $\mathrm{Mn}$ center by adopting a monodentated coordination fashion. Two Mn(II) are both five-coordinated with a trigonal bipyramid geometry. Mn1 is coordinated by three carboxyl groups from three different L ligands, and two N1 and N4 from two mi ligands. The $\mathrm{Mn}-\mathrm{O} / \mathrm{N}$ lengths are in normal, which are similar with those reported polymers [13-15]. The two symmetric Mn centers are bridging by carboxylate groups with 
Mn...Mn distances of $3.88 \AA$. These chains can be taken as $\left[\mathrm{Mn}_{2}\left(\mathrm{CO}_{2}\right)_{4}\right]$ subunits, which are cross-linked by the mi linlgands into a 1D double stranded chain, as shown in Figure 2.

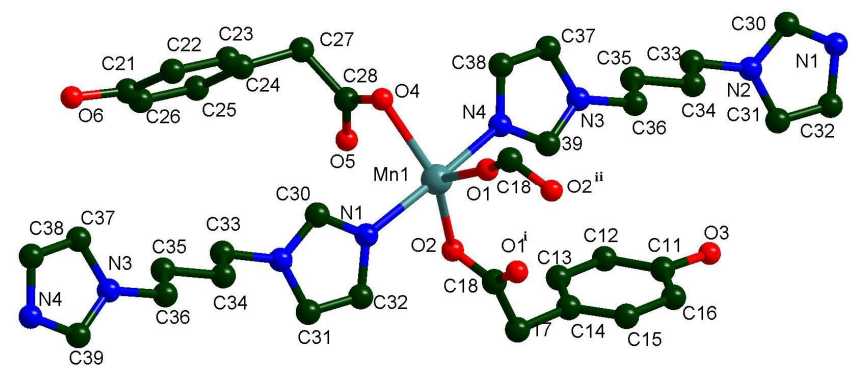

Figure 1. The coordination geometries of the metal centers and the ligands geometries in $\mathbf{1}$. Displacement ellipsoids are drawn at the $30 \%$ probability level and $\mathrm{H}$ atoms are omitted for clarity (symmetric code (i) x, y, z; (ii) -x, -y, -z).

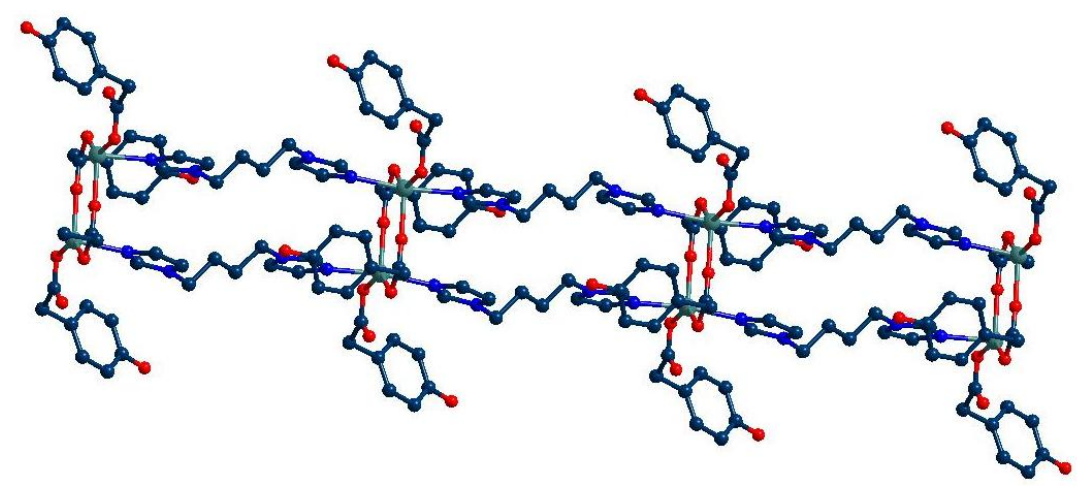

Figure 2. View of the 1D packing chain directing by mi linkers.

The one-dimensional structure of $\mathbf{1}$ is stabilized by multiple hydrogen-bonding interactions involving carboxylate ligand, free water molecule and phenolic group. The donor-acceptor bond distances, $\mathrm{O}-\mathrm{H} \cdots \mathrm{O}$, are in the range of $2.680(8)-3.129(7) \AA$ (Table 3). These hydrogen bonds link different 1D double-stranded chains to form a 2D network.

Table 3. Hydrogen bonding distances $(\AA)$ and angles $\left(^{\circ}\right)$ for $\mathbf{1}$.

\begin{tabular}{|l|l|l|c|}
\hline O3 $\cdots \mathrm{O} 5$ & 2.6813 & $\mathrm{O} 1 \mathrm{~W} \cdots \mathrm{O} 4$ & 2.8126 \\
\hline O6 $\cdots \mathrm{O} 1 \mathrm{~W}$ & 2.6925 & $\mathrm{O} 1 \mathrm{~W} \cdots \mathrm{O} 3$ & 3.1312 \\
\hline $\mathrm{O} 3-\mathrm{H} 1 \cdots \mathrm{O} 5$ & $177.1(2)$ & $\mathrm{O} 1 \mathrm{~W}-\mathrm{H} 1 \mathrm{~W} 1 \cdots \mathrm{O} 4$ & $169.4(2)$ \\
\hline O1W-H1W2 $\cdots \mathrm{O} 3$ & $153.9(11)$ & $\mathrm{O} 6-\mathrm{H} 2 \cdots \mathrm{O} 1 \mathrm{~W}$ & $167.3(7)$ \\
\hline
\end{tabular}

FTIR, TGA and XPRD analysis of complexes 1 and $\mathbf{2}$

The compound shows a broad band centered around $3200 \mathrm{~cm}^{-1}$, which may be attributed to the $\mathrm{O}-\mathrm{H}$ stretching frequency of the water. Specifically, asymmetric stretching vibration $v\left(\mathrm{COO}^{-}\right)$ appear around $1600 \mathrm{~cm}^{-1}$ for 1 , respectively, and the symmetric stretching vibration $v\left(\mathrm{COO}^{-}\right)$are observed $1430 \mathrm{~cm}^{-1}$ and $1320 \mathrm{~cm}^{-1}$. For the title complex, the difference between the asymmetric 
and symmetric stretches, $\Delta v_{\text {as }\left(\mathrm{COO}^{-}\right)^{-}} v_{\mathrm{s}\left(\mathrm{COO}^{-}\right)}$, are on the order of $200 \mathrm{~cm}^{-1}$ indicating that carboxyl groups are coordinated to the metal in a mono-dentate and bidentate modes [22], consistent with the observed X-ray crystal structure of $\mathbf{1}$.

To study the stability of the polymer, thermogravimetric analyses (TGA) of complex 1 was performed. The TGA diagram of 1 showed two weight loss steps. The first weight loss began at $35^{\circ} \mathrm{C}$ and completed at $165^{\circ} \mathrm{C}$. The observed weight loss of $3.3 \%$ is corresponding to the loss of the water molecule (calcd 3.2\%). The second weight loss occurs in the range $250-600{ }^{\circ} \mathrm{C}$, which can be attributed to the elimination of $\mathrm{L}$ and mi ligands.

Additionally, to confirm the phase purity and stability of compound 1, the original sample was characterized by X-ray powder diffraction (XRPD). Although the experimental patterns have a few unindexed diffractions lines and some are slightly broadened in comparison to those simulated from single-crystal models, it can still to be considered that the bulk synthesized materials and as-grown crystal are homogeneous for compound $\mathbf{1}$.

\section{Magnetic property}

The $\chi_{\mathrm{M}} T$ value for the complex 1 was $8.51 \mathrm{~cm}^{3} \mathrm{~K} \mathrm{~mol}^{-1}$ at $300 \mathrm{~K}$, and this is close to the spinonly value $\left(8.75 \mathrm{~cm}^{3} \mathrm{~K} \mathrm{~mol}^{-1}\right)$ for the dimer of $S=5 / 2$ ions (Figure 3$)$. When decreasing the temperature, a significant drop was observed below $50 \mathrm{~K}$, and reached to $0.79 \mathrm{~cm}^{3} \mathrm{~K} \mathrm{~mol}^{-1}$ at 2 $\mathrm{K}$. This indicates an anti-ferromagnetic interaction between manganese(II) ions. In the $\chi_{\mathrm{M}}$ versus $T$ plot, a maximum was observed at $T_{\max }=6-7 \mathrm{~K}$, and this corresponds to the $2 J$ value of -1.45 $-1.70 \mathrm{~cm}^{-1}\left(\mathrm{H}=-2 J \mathrm{~S}_{\mathrm{A}} \cdot \mathrm{S}_{\mathrm{B}}\right)$, judging from the relationship $|2 J| / k T_{\max }=0.347$. Cryomagnetic data were analyzed according to the standard equation for $S_{\mathrm{A}}=S_{\mathrm{B}}=5 / 2$ system (equations 2, 3) [23].

$\chi_{M}=\frac{2 N g^{2} \beta^{2}}{k T} \frac{e^{x}+5 e^{3 x}+14 e^{6 x}+30 e^{10 x}+55 e^{15 x}}{1+3 e^{x}+5 e^{3 x}+7 e^{6 x}+9 e^{10 x}+11 e^{15 x}}$,

with

$x=2 J / k T$.

The optimized parameters were $2 J=-1.48 \mathrm{~cm}^{-1}$ and $g=2.03 \mathrm{~cm}^{-1}$. The obtained $2 J$ value is consistent with the value obtained based on the $T_{\max }$ value, and the antiferromagnetic interaction was observed between manganese(II) ions.

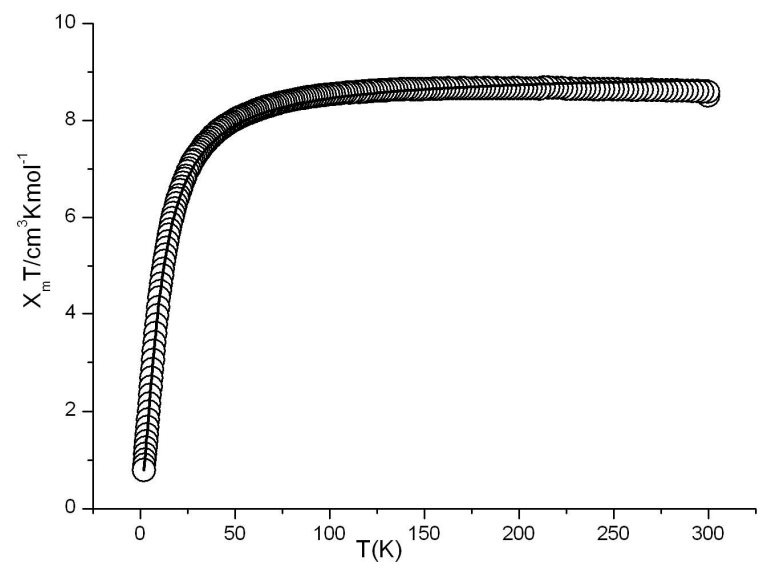

Figure 3. Plots $\chi_{M} T$ versus $T$ for $\mathbf{1}$, solid lines represent fits to the data.

Bull. Chem. Soc. Ethiop. 2016, 30(1) 
The parameter $\Phi$ bend of $\mathrm{Mn}-\mathrm{O}-\mathrm{C}-\mathrm{O}-\mathrm{Mn}$ is very important. The larger $\Phi$ bend could generate a larger decrease in $-J$ because of reduced overlap of the $\mathrm{d}_{\mathrm{x} 2-\mathrm{y} 2}$ orbital with the $2 \mathrm{p}_{\mathrm{x}}$ carboxylate oxygen orbital in the symmetric HOMO [24]. In the case of 1 , $\Phi$ bend is $5.1(8)^{\circ}$, which is slightly larger than that of $\left[\mathrm{Mn}_{3}(\mathrm{~L})(\mathrm{DMA}) \cdot 2 \mathrm{DMA}\right] \quad\left(\mathrm{H}_{6} \mathrm{~L}=\right.$ hexa[4(carboxyphenyl)oxamethyl]-3-oxapentane acid) [25]. Liu et al. reported [Mn(hfac)2NIT(Ph-m$\mathrm{OPh})$ ] consists of a 1-D chain with $\mathrm{Mn}(\mathrm{II})$ bridged by NIT(Ph-m-OPh) [26], which exhibits an intramolecular antiferromagnetic interaction between $\mathrm{Mn}(\mathrm{II})$ and NIT(Ph-m-OPh). A dinuclear complex of manganese(II), $\left[\mathrm{Mn}(\mathrm{L})(\mu-1,5-\mathrm{dca})\left(\mathrm{CH}_{3} \mathrm{OH}\right)\right]_{2}$, with dicyanamide anion and (E)-3hydroxy-N'- (pyridin-2-ylmethylene)-2-naphthohydrazide (HL) was synthesized. It also shows an antiferromagnetic interaction between adjacent manganese ions in the dimer [27]. These compounds and the title compound also have an antiferromagnetic interaction between adjacent manganese ions due to similar parameter $\Phi$ bend of $\mathrm{Mn}-\mathrm{O}-\mathrm{C}-\mathrm{O}-\mathrm{Mn}$ [28].

\section{Hirshfeld surface analysis}

The Hirshfeld surfaces for the complex $\mathbf{1}$ is illustrated in Figure 4 showing surfaces that have been mapped over a $d_{\text {norm }}$ range of -0.5 to $1.5 \AA$, shape index ( -1.0 to $1.0 \AA$ ) and curvedness ( -4.0 to 0.4 $\AA$ ). The surfaces are shown as transparent to allow visualization of all the atoms of the molecule around which they were calculated. The weak interaction information discussed in X-ray crystallography section is summarized effectively in the spots, with the large circular depressions (deep red) visible on the $d_{\text {norm }}$ surfaces indicative of hydrogen bonding contacts. The dominant interactions between $\mathrm{O}-\mathrm{H} \cdots \mathrm{O}$ for the manganese compound can be seen in Hirshfeld surface plots as the bright red shaded area in Figure 6.

The fingerprint plots for $\mathbf{1}$ are presented in Figure 5. The $\mathrm{O} \cdots \mathrm{H}$ and $\mathrm{N} \cdots \mathrm{H}$ intermolecular interactions appear as two distinct spikes of almost equal lengths in the 2D fingerprint plots in the region $2.03 \AA<\left(d_{\mathrm{e}}+d_{\mathrm{i}}\right)<2.47 \AA$ as light sky-blue pattern in full fingerprint $2 \mathrm{D}$ plots. Complementary regions are visible in the fingerprint plots where one molecule acts as a donor $\left(d_{\mathrm{e}}\right.$ $\left.>d_{\mathrm{i}}\right)$ and the other as an acceptor $\left(d_{\mathrm{e}}<d_{\mathrm{i}}\right)$. The fingerprint plots can be decomposed to highlight particular atom pair close contacts. This decomposition enables separation of contributions from different interaction types, which overlap in the full fingerprint. The proportions of $\mathrm{O} \cdots \mathrm{H}$ for complex $\mathbf{1}$ is $22.9 \%$ of the total Hirshfeld surface while $\mathrm{N} \cdots \mathrm{H}$ interactions for complex $\mathbf{1}$ constitutes $4.1 \%$ of the total Hirshfeld surface.
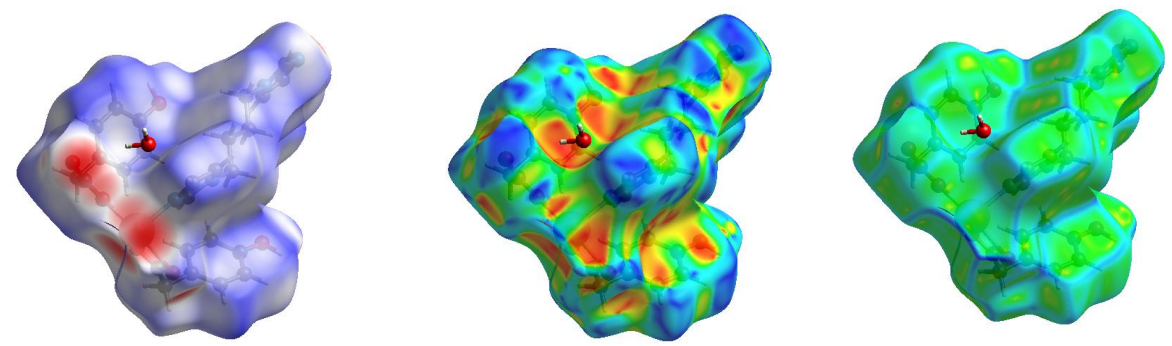

Figure 4. Hirshfeld surfaces mapped with $d_{\text {norm}}$, shape index and curvedness for the $\mathbf{1}$.

\section{Atoms in molecule (AIM) calculations}

To confirm the presence of $\mathrm{Mn} \cdots \mathrm{Mn}$ bond critical points (bcp) were calculated for for the dimer (Figure 6) by using the Atoms in Molecules theory [29]. The bond critical points though observed between the $\mathrm{Mn}$ and $\mathrm{O}$ in dimer but no $\mathrm{Mn} \cdots \mathrm{Mn}$ interaction was observed. The absence of bond 
critical points between two Mn centers undoubtedly verifies the antiferromagnetic interaction which was observed at or below $50 \mathrm{~K}$.
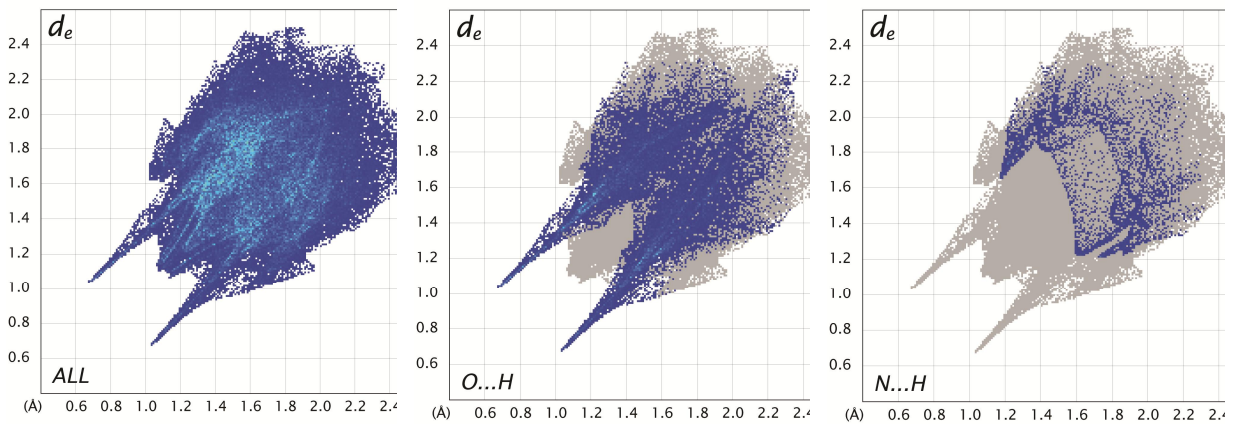

Figure 5. Fingerprint plots Full (left), resolved into $\mathrm{O} \cdots \mathrm{H}$ (middle) and resolved into $\mathrm{N} \cdots \mathrm{H}$ (right) for the compound 1 .

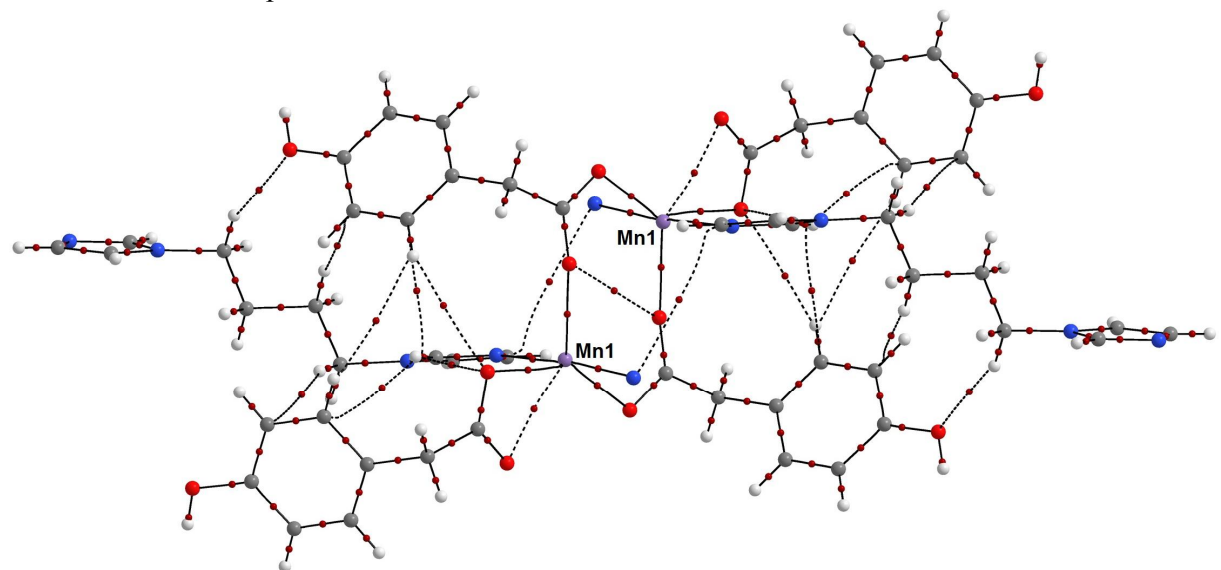

Figure 6. Molecular graph for complex 1 computed at B3LYP/6-311G** level of theory showing various bond critical points.

\section{CONCLUSION}

In conclusion, we have presented synthetic strategy that successfully achieved one dinuclear Mn-based complex 1, which shows the antiferromagnetic interaction between the Mn(II) ions. The observed antiferromagnetic interaction has been corroborated using AIM theory which also establishes that no $\mathrm{Mn} \cdots \mathrm{Mn}$ interaction is operating within the molecule. This observation indicates that ligand engineering can promote $\mathrm{Mn} \cdots \mathrm{Mn}$ interaction which can eventually lead to significant drop in the ferromagnetism even at the elevated temperature.

\section{ACKNOWLEDGMENTS}

The authors acknowledge financial assistance from Sichuan University of Science and Engineering (nos. 2014PY01, 2015PY03 and 2015RC26), and the Education Committee 
of Sichuan Province (nos. 12ZA090, 14ZB0220, 15ZB0222, 15ZB0214), and greater contribution from Prof. Hiroshi Sakiyama. AK is grateful to DST, New Delhi for financial support in the form of project no. SB/FT/CS-018/2012.

\section{REFERENCES}

1. Chen, B.L.; Xiang, S.C.; Qian, G.D. Acc. Chem. Res. 2010, 43, 111.

2. Carlucci, L.; Ciani, G.; Proserpio, D.M. Coord. Chem. Rev. 2003, 246, 247.

3. Raymond, J.; Blankenship, R.E. Coord. Chem. Rev. 2008, 252, 377.

4. Sun, C.Y.; Gao, S.; Jin, L.P. Eur. J. Inorg. Chem. 2006, 12, 2411.

5. Liu, J.Q.; Wang, Y.Y.; Liu, P.; Dong, Z.; Shi, Q.Z.; Batten, S.R. CrystEngComm. 2009, 11, 207.

6. Ededet, E.A.; Ayimele, G.A.; Wang, Z.X.; Chongwain, P.T.; Iniama, G.E.; Offiong, O.E.; Nfor, E.N. Bull. Chem. Soc. Ethiop. 2011, 25, 53.

7. Tabatabaee, M.; Mohamadinasab, R.; Ghaini, K.; Khavasi, H.R. Bull. Chem. Soc. Ethiop. 2010, 24, 401.

8. Du, M.; Li, C.P.; Guo, J.H. CrystEngComm. 2009, 11, 1536.

9. Wuest, J.D. Chem. Commun. 2005, 2005, 5830.

10. MacGillivray, L.R. J. Org. Chem. 2008, 73, 3311.

11. Glerup, J.; Goodson, P.A.; Hodgson, D.J.; Jensen, N.; Michelsen, K. Bull. Chem. Soc. Ethiop. 2004, 18, 17.

12. Idbal, M.; Ahmad, I.; Ali, S.; Muhammad, N.; Ahmed, S.; Sohail, M. Polyhedron 2013, 50, 524.

13. Zhuo, X.; Xu, J.G.; Zhang, L.; Liu, C. Chinese J. Struct. Chem. 2010, 29, 1743.

14. Sheldrick, G.M. SHELXL-97: Program for Structure Determination and Refinement, University of Göttingen: Göttingen; 1997.

15. Spackman, M.A.; McKinnon, J.J. CrystEngComm. 2002, 4, 378.

16. McKinnon, J.J.; Mitchell, A.S.; Spackman, M.A. Chem-Eur. J. 1998, 4, 2136.

17. Rohl, A.L.; Moret, M.; Kaminsky, W.; Claborn, K.; McKinnon, J.J.; Kahr, B. Cryst. Growth Des. 2008, 8, 4517.

18. Parkin, A.; Barr, G.; Dong, W.; Gilmore, C.J.; Jayatilaka, D.; McKinnon, J.J.; Spackman, M.A.; Wilson, C.C. CrystEngComm. 2007, 9, 648.

19. Wolff, S.K.; Greenwood, D.J.; McKinnon, J.J.; Jayatilaka, D.; Spackman, M.A. Crystal Explorer 2.0, University of Western Australia: Perth, Australia; 2007.

20. Koenderink, J.J.; van Doorn, A.J. Image Vision Comput. 1992, 10, 557.

21. Maheshwary, S.; Patel, N.; Sathyamurthy, N.; Kulkarni, A.D.; Gadre, S.R. J. Phys. Chem. A 2001, 105, 10525.

22. Nakamoto, K. Infrared and Raman Spectra of Inorganic and Coordination Compounds, 5th ed., Wiley Interscience: New York; 1997.

23. Kahn, O. Molecular Magnetism, VCH: Weinheim; 1993.

24. Manson, J.L; Ressouche, E.; Miller, J.S. Inorg. Chem. 2000, 39, 1135.

25. Landee, C.P.; Turnbull, M.M. J. Coord. Chem. 2014, 67, 375.

26. Qin, X.Y.; Xiong, G.; Liao, D.Z.; Ma, Y.; Gao, P.; Sun, X.L.; Liu, P. J. Coord. Chem. 2012, $65,2683$.

27. Bikas, R.; Hoeesini-Monfared, H.; Sieron, L. Gutierrez, A. J. Coord. Chem. 2013, 66, 4023.

28. Glerup, J.; Goodson, P.A.; Hodgson, D.J.; Michelsen, K.; Rychlewska, R.; Weihe, H. Bull. Chem. Soc. Ethiop. 2000, 14, 128.

29. Bader, R.F.W. In Atoms in Molecules: A Quantum Theory, Oxford University Press: New York; 1990. 